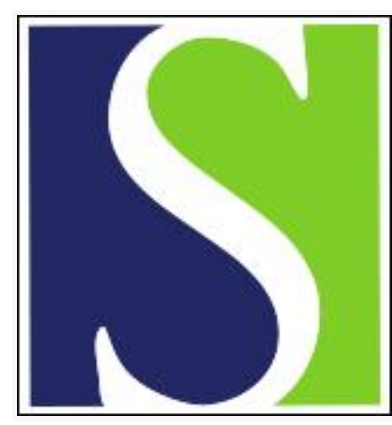

Scand J Work Environ Health 1987;13(4):385-388

https://doi.org/10.5271/sjweh.2040

Issue date: Aug 1987

New techniques for the diagnosis of carpal tunnel syndrome.

by Bleecker ML, Agnew J

Affiliation: Department of Neurology, Francis Scott Key Medical Center, Baltimore, Maryland 21224.

This article in PubMed: www.ncbi.nlm.nih.gov/pubmed/3324318

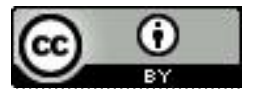




\title{
New techniques for the diagnosis of carpal tunnel syndrome
}

\author{
by Margit L Bleecker, MD, $\mathrm{PhD},{ }^{1,2}$ Jacqueline Agnew, $\mathrm{PhD}^{2}$
}

\begin{abstract}
BLEECKER ML, AGNEW J. New techniques for the diagnosis of carpal tunnel syndrome. Scand J Work Environ Health 13 (1987) 385-388. An overview is presented of the assessment of carpal tunnel syndrome (CTS) in manual workers, with emphasis on the distinction from other compression syndromes and from diffuse polyneuropathy. Clinical examinations, electrodiagnostic studies, the determination of vibration threshold, thermography, and, recently, computerized tomography have been used. This last-mentioned method has also been employed in a study indicating that a subgroup of the general population may possess a risk factor, small carpal canal, associated with the development of CTS.
\end{abstract}

Key terms: clinical findings, computerized tomography, electrodiagnostic studies, thermography, vibration threshold.

A simple definition of carpal tunnel syndrome (CTS) is a disorder resulting from compression or irritation of the median nerve as it passes into the hand between the carpal bones and the transverse carpal ligament with subsequent discomfort and impaired use of the hand. The carpal canal is formed by the concave arch of the carpal bones and is roofed by the transverse carpal ligament. These structures form a rigid compartment through which nine tendons and the median nerve must pass.

\section{Clinical presentation}

Unless associated with direct trauma, the onset of symptoms of CTS is usually nocturnal and insidious. The pain in the hand may initially be described as episodic tingling and numbness with gradual progression to more severe symptoms referred to as burning, aching, pricking, or as a painful numbness in the fingers and deep in the palm. With the pain and tingling there is a subjective feeling of uselessness in the fingers, which are sometimes described as feeling swollen, even though, on inspection, little swelling is apparent. Many patients will have accompanying pain in the forearm, sometimes reaching the shoulder and described as a dull aching pain felt deeply in the limb.

Color changes in the fingers have been described, particularly with exposure to cold, but they are not related to the attacks of pain. In addition excessive sweating and mild degrees of edema are related to the vasomotor imbalance known to occur in CTS (1).

\footnotetext{
1 Johns Hopkins Medical Institutions, Department of Neurology, Francis Scott Key Medical Center, Baltimore, Maryland, United States.

2 Environmental Health Science, Division of Occupational Medicine.
}

Reprints requests to: Dr ML Bleecker, Johns Hopkins Medical Institutions, Department of Neurology, Francis Scott Key Medical Center, 4940 Eastern Avenue, Baltimore, MD 21224 , USA.
As CTS progresses, the nocturnal pain and tingling may begin to wake the patient after a few hours of sleep. Relief may be obtained by hanging the arm out of bed or shaking or rubbing the hand, but as symptoms increase, patients often get out of bed and walk about until the symptoms have eased. At this stage episodic tingling may develop during the day, but the associated pain in the arm occurs less often during the day than at night. In addition to sensory symptoms, there may be clumsiness and difficulty in performing certain tasks, such as unscrewing bottle tops, turning a key, or crocheting.

Objective changes in sensation and strength may appear in the hand, but some patients may suffer severe attacks of pain for many years without developing abnormal neurological signs. Sensory signs are best sought in the fingertips, where impairment is, as a rule, more pronounced. Occasionally, instead of decreased sensation, there is an overreaction to cutaneous stimuli in the median innervated digits. Isolated thenar wasting or sensory impairment in the distribution of one of the lateral three digital nerves may be the presenting feature of median nerve lesions at the wrist. Mild weakness of the abductor pollicis brevis or of the opponens pollicis muscle is frequently present with no visually apparent atrophy. Manual pressure over the flexor aspect of the wrist or prolonged hyperextension or hyperflexion of this joint may produce sensory symptoms (Phalen's sign). Tinel's sign, consisting of shocklike pain and tingling elicited by percussion of the median nerve at the wrist, is a less common finding than often thought (10).

\section{Electrodiagnostic studies}

Although CTS abnormalities are more likely to be found in nerve conduction studies in the presence of a defect found in a clinical examination, a significant number of patients with typical CTS symptoms have 
no abnormalities other than those detected in electrodiagnostic testing.

Compression of the median nerve in the carpal tunnel produces slow nerve conduction, a block of nerve conduction, or axonal destruction. For many years, tests for this syndrome have included measuring the time for a nerve impulse to travel through the median nerve from the wrist to the abductor pollicis brevis muscle (distal motor latency) and from the index finger to the wrist with orthodromic stimulation or from the wrist to the index finger with antidromic stimulation (distal sensory latency). The sensitivity of these measurements is limited because of the inclusion of the relatively normal segment of the nerve distal to the carpal ligament.

Several newer techniques are especially helpful in the early diagnosis of CTS. Recently the conduction time of sensory fibers has been measured in the segment across the carpal tunnel. With palmar stimulation the median nerve is stimulated distal to the carpal ligament in the midpalm, and the sensory nerve action potential is recorded $8 \mathrm{~cm}$ proximally, just above the wrist. The median palmar latency is compared with the ulnar palmar latency measured over the same distance. Prolonged median palmar latencies or latencies relatively greater than the ulnar value suggest CTS. Palmar stimulation identifies approximately $95 \%$ of patients with CTS (5). Prolongation of both the ulnar and median distal latencies suggests low hand temperature or peripheral neuropathy.

Occasionally it is helpful to determine the site of maximal conduction delay in the distal median nerve by stimulation at multiple sites along the nerve. Latencies are measured from the index finger to the palm, from the palm to the wrist, and from the wrist to the forearm. These measurements allow one to distinguish between CTS and peripheral neuropathy affecting the median nerve in which the maximal slowing will be found in the segment from the palm to the finger rather than across the carpal tunnel.

Stimulation of the median nerve from the palm to the wrist in $1-\mathrm{cm}$ increments in patients with CTS has shown that, in $53 \%$ of affected nerves, there is a sharply localized latency increase across a $1-\mathrm{cm}$ segment, most commonly $2-4 \mathrm{~cm}$ distal to the origin of the tranverse carpal ligament. This slowing appears to correspond to the region of the distal edge of a carpal ligament. In the remaining $47 \%$ of nerves, the slowing is distributed more diffusely across the carpal tunnel (12). Median nerves are tested bilaterally in CTS patients because of the high incidence of conduction abnormalities in the asymptomatic extremities of CTS patients. Other nerves are tested because of the occasional patient who presents a diffuse neuropathy with CTS.

Section of the transverse carpal ligament in CTS usually results in improvement in both clinical symptoms and the long median nerve distal latency. However, the latency abnormality often does not return to normal (14). Needle-electrode examination of the thenar eminence is uncomfortable and less sensitive in CTS than are routine nerve conduction studies (16).

Calculation of residual latency, only recently reported (13), is important for eliminating the effect of variability in nerve conduction velocity upon distal latency measurements. Residual latencies hold constant into the ninth decade in contrast to conduction velocities which continue to slow with increasing age. With a recent onset of CTS, prolonged residual latencies may be the only abnormality.

It has been recently demonstrated that median innervated lumbrical muscles are relatively spared in advanced CTS. Therefore, increased diagnostic sensitivity for CTS is obtained when comparison is made to the involved abductor pollicus brevis. In CTS, the distal latency to the abductor pollicis brevis is greater than the distal latency to the second lumbrical after stimulation of the median nerve at the wrist (15).

\section{Vibration threshold}

The large myelinated fibers are decreased in number in the median palmar digital nerves in CTS (17). Abnormalities in this population of axons should be reflected in vibration sensation, which is carried by this fiber population. Abnormal vibratory perception in the median inervated index finger in comparison to the perception in the ulnar innervated ipsilateral little finger has been reported as the earliest nonprovocative sign of CTS. Dellon (7) found an abnormal perception of vibratory stimuli in $72 \%$ of patients with a history compatible with CTS.

A practical tool for determining vibration perception threshold is a tactile stimulator. When the maximum sensitivity occurs at $200-300 \mathrm{~Hz}$, the pacinian corpuscle, a rapidly adapting mechanoreceptor, is stimulated. Studies show that the difference in the vibratory threshold between the involved digit II and the uninvolved digit $\mathrm{V}$ is significantly elevated in CTS (3). Threshold tests of sensibility (pressure monofilaments and vibration) correlate accurately with symptoms of nerve compression (9).

\section{Thermography}

Liquid crystal thermography has only recently been applied in the study of CTS. It appears to be sensitive in detecting asymmetries in heat distribution (8). Liquid crystal thermographs probably reflect vasomotor instability resulting from compromise of the sympathetic nerves, which are carried in the median nerve. As a noninvasive method for diagnosing and following CTS, this method may have potential, but further studies are still needed to determine whether this technique is identifying only a subpopulation of individuals with CTS or if these temperature changes are a universal finding in this disorder. 


\section{Computerized tomography of the carpal canal}

Characterization of overall hand size was tabulated by Armstrong \& Chaffin (2) in a group of workers with CTS and a group of referents. Anthropometric data collected from surface measurement of the hand and wrist, including external measurements of hand length, palm width, wrist width, and wrist thickness and the internal dimension of the third metacarpal length, were selected for the characterization of overall hand size. No significant differences between the two groups were found. Even when several internal measurements were made from radiographs to estimate the carpal angle, which is the curvature of the carpal bones, no association was found between hand shape and CTS.

Measurements of the cross-sectional area of the carpal tunnel reveal a narrow carpal tunnel in females with CTS (11). Rather than using vertical roentgenograms of the bony boundaries of the carpal groove, Dekel et al (6) demonstrated carpal canal stenosis in idiopathic CTS with manual digitization of computerized tomograms through the carpal canal. Both of these studies examined only females with CTS since idiopathic CTS is generally associated with females between 40 and 60 years of age.

Carpal canal stenosis may be congenital or acquired by trauma or arthritis, but, as an etiologic factor in occupationally related CTS, it has been given little attention. Carpal canal size as a predictor for the development of this entrapment syndrome was studied in a group workers (4). Seven of 14 electricians had symptomatic carpal tunnel syndrome. Workers classified as having clinically apparent CTS had appropriate responses on the symptom questionnaire and electrophysiological findings of CTS. Subclinical cases included asymptomatic workers with physiological evidence of the syndrome. "Normal" subjects had no clinical or physiological evidence of nerve entrapment.

Computerized tomography was performed on a GE $8800 \mathrm{CT} / \mathrm{T}$ scanner. A scout film was obtained to insure proper alignment. The first tomographic slice was taken at the palmar crease, followed by $5-\mathrm{mm}$ contiguous slices through the carpal canal to the metacarpal level. At each level, a cursor in the scanner outlined the circumference of the carpal canal. The computer calculated canal area for each level. The depth at the median nerve and width of the canal were measured, as well as the thickness of the transverse carpal ligament. Affected workers had a mean crosssectional area of $1.75+0.21 \mathrm{~cm}^{2}$; the reference values were $2.23+0.15 \mathrm{~cm}^{2}(\mathrm{P}<0.05)$. Individuals with a subclinical form of CTS had an area of $1.93+0.22 \mathrm{~cm}^{2}$, similar to that of the symptomatic group. Wrist circumference was not a reliable predictor of the smallest carpal canal area. Unusual bony and soft tissue structures within the carpal canal were easily identified with the computerized tomography.

In a second study, carpal canal size was examined in eight men with an age distribution (20-60 years) comparable to that of the aforementioned study, a normal physical examination of the upper extremities, normal nerve conduction studies, and no exposure to occupations or hobbies which involved repeated and forceful movement of flexor tendons through the carpal tunnel. The range of the smallest carpal canal size per wrist was identical to that found among the electricians with a median of 1.75 (range 1.34 2.01) $\mathrm{cm}^{2}$. These results suggest that a subgroup of the general population may possess a risk factor, small carpal canal, associated with the development of CTS. However, with no exposure to known ergonomic stresses or other risk factors associated with the development of CTS, these individuals may remain free of symptoms secondary to median nerve compression in the carpal canal.

Computerized tomography may reveal spaceoccupying structures within the canal that are not apparent by external examination. Accumulations of fat, muscle insertions within the canal, bony abnormalities (such as a protruding head of the capitate), fractures, tenosynovitis (causing adhesions between the long flexor tendons), and a thickened transverse carpal ligament are all features that compromise the area in the canal needed for the long flexor tendons and the median nerve. Wrist circumference has been a weak predictor for CTS because it does not reflect these canal anomalies. Body weight affects wrist circumference, but does not alter the size of the canal.

An association between body weight and the quantity of fat in the canal was not found. Further evaluation is needed to establish the significance of the relationship between long bones, as measured by height, and the small carpal bones composing the canal.

Computerized tomography has been used on our clinic patients with idiopathic CTS in the nondominant hand. It revealed carpal canal stenosis. This etiology should be considered when symptoms are relieved by splinting and recur whenever the splint is removed. Establishing carpal canal stenosis as the etiology may eliminate months of fruitless conservative therapy before release surgery is performed.

\section{Reference}

1. Aminoff MJ. Involvement of peripheral vasomotor fibres in Carpal Tunnel Syndrome. J Neurol Neurosurg Psychiatry 42 (1979) 649-655.

2. Armstrong TJ, Chaffin DB. Carpal tunnel syndrome selected personal attributes. J Occup Med 21 (1979) $481-486$.

3. Bleecker M. The optacon: A new screening device for peripheral neuropathy. In: Advances in the biosciences: Neurobehavioral methods in occupational health. Pergaman Press, Oxford 1983, pp 41-46.

4. Bleecker ML, Bohlman M, Moreland R, Tipton A. Carpal tunnel syndrome: Role of carpal canal size. Neurology 35 (1985) 1599-1604.

5. Buchthal F, Rosenfalck A. Sensory conduction from digit to palm and from palm to wrist in the carpal tunnel syndrome. J Neurol Neurosurg Psychiatry 34 (1971) 243.

6. Dekel S, Papaioannori T, Rushworth G, Coates R. 
Idiopathic carpal tunnel syndrome caused by carpal stenosis Br Med J 1 (1980) 1297-1299.

7. Dellon AL. Clinical use of vibratory stimuli to evaluate peripheral nerve injury and compression neuropathy. Plast Reconstr Surg 15 (1980) 466-470.

8. Gateless D. The diagnosis of carpal tunnel syndrome in pregnant women using liquid crystal thermography. Neurology 33 (1983) 240.

9. Gelberman RH. Sensibility testing in peripheral nerve compression syndrome. J Bone Jt Surgery 65A (1983) 632-638.

10. Gelmars $\mathbf{H}$. The significance of Tinel's sign in the diagnosis of carpal tunnel syndrome. Acta Neurochir 49 (1979) 255-258.

11. Gelmars H. Primary carpal tunnel stenosis as a cause of entrapment of the median nerve. Acta Neurochir 55 (1981) 317-320.

12. Kimura J. The carpal tunnel syndrome: Localization of conduction abnormalities within the distal segment of the median nerve. Brain 102 (1979) 619-635.

13. Kraft GH. Median nerve residual latency normal value and use in diagnosis of carpal tunnel syndrome. Arch Phys Med Rehabil 64 (1983) 221-226.

14. LeQuesne PM, Casey EB. Recovery of conduction velocity distal to a compressive lesion. $J$ Neurol Neurosurg Psychiatry 37 (1974) 1346-1351.

15. Logigian EL, Busis NA, Berger BT, Shahani RR, Young. Sparing of the lumbrical muscles in carpal tunnel syndrome. Neurology 35 (1985) 35-68.

16. Thomas JF, Lambert EH, Czeuz KA. Electrodiagnostic aspects of the carpal tunnel syndrome. Arch Neurol 16 (1967) 635-641.

17. Thomas PK, Fullerton PM. Nerve fiber size in the carpal tunnel syndrome. J Neurol Neurosurg Psychiatry 26 (1963) 520-527. 